EUROPEAN ORGANIZATION FOR NUCLEAR RESEARCH

Laboratory for Particle Physics

Divisional Report

CERN LHC/2000-5

(DLO)

\title{
NEUTRALIZATION PRINCIPLES \\ FOR THE EXTRACTION AND TRANSPORT OF ION BEAMS
}

\author{
H. Riege
}

The strict application of conventional extraction techniques of ion beams from a plasma source is characterized by a natural intensity limit determined by space charge. The extracted current may be enhanced far beyond this limit by neutralizing the space charge of the extracted ions in the first extraction gap of the source with electrons injected from the opposite side. The transverse and longitudinal emittances of a neutralized ion beam, hence its brightness, are preserved. Results of beam compensation experiments, which have been carried out with a laser ion source, are resumed for proposing a general scheme of neutralizing ion sources and their adjacent low-energy beam transport channels with electron beams. Many technical applications of high-mass ion beam neutralization technology may be identified: the enhancement of ion source output for injection into high-intensity, low-and high-energy accelerators, or ion thrusters in space technology, for the neutral beams needed for plasma heating of magnetic confinement fusion devices, and for future accelerator-driven systems for fusion, fission and nuclear waste incineration.

Published in Nuclear Instruments and Methods in Physics Research

Section A, vol. 451, Issue 2

Administrative Secretariat

LHC Division

CERN

CH - 1211 Geneva 23
Geneva, Switzerland

1 September 2000 


\title{
Neutralization principles for the extraction and transport of ion beams
}

\author{
H. Riege ${ }^{\mathrm{a}}$ \\ a CERN LHC Division, CH-1211 Geneva 23, Switzerland
}

The strict application of conventional extraction techniques of ion beams from a plasma source is characterized by a natural intensity limit determined by space charge. The extracted current may be enhanced far beyond this limit by neutralizing the space charge of the extracted ions in the first extraction gap of the source with electrons injected from the opposite side. The transverse and longitudinal emittances of a neutralized ion beam, hence its brightness, are preserved. Results of beam compensation experiments, which have been carried out with a laser ion source, are resumed for proposing a general scheme of neutralizing ion sources and their adjacent low-energy beam transport channels with electron beams. Many technical applications of high-mass ion beam neutralization technology may be identified: the enhancement of ion source output for injection into high-intensity, low- and high-energy accelerators, for ion thrusters in space technology, for the neutral beams needed for plasma heating of magnetic confinement fusion devices, and for future accelerator-driven systems for fusion, fission and nuclear waste incineration.

\section{Introduction}

Classically, ion beams are extracted from a plasma source and injected for further transport into vacuum. In nature we observe that free charges of opposite polarity tend to attract each other and currents to cancel (Faraday's induction law). The mass of neutral objects which can be transported through space is unlimited. Owing to the tendency to self-neutralization charged particle beam neutralization can technically be achieved much easier than, for example, the acceleration of a charged particle beam.

In the past a few cases of successful neutralization schemes for low-energy charged particle beams with particle clouds or beams of opposite polarity or with a low-density plasma have been demonstrated $[1,2,3]$. A new method of neutralizing heavy-ion beams with intense pulsed, low-energy electron beams 
generated by ferroelectrically-driven electron guns has recently been experimentally applied to the extraction from a laser ion source and is reported in Refs. [4-7]. In various technical fields beam compensation methods have been successfully applied for many years. One example is the development of neutralized ion thrusters in the space industry for satellite and spacecraft propulsion, where the ejected ion beams are charge and current neutralized with electrons exiting from a thermionically-generated electron cloud or from a plasma reservoir $[8,9]$. Other cases are the plasma heating of magnetic confinement fusion tokomacs achieved by the injection of neutralized ion beams, and the use of plasma-neutralized ion sources for efficient ion implantation into the large-area surface layers of three-dimensional objects [10]. However, it should be noted that in all these examples of beam neutralization usually the ion beams drifting outside the source are neutralized, whereas the ions are not charge-neutralized during the extraction phase from the ion source plasma.

In accelerators for high-energy physics the beam compensation of ion sources is a promising method for raising output current density above the Langmuir-

Child limit. Furthermore, the beam quality is also preserved, as the emittance of neutralized beams is not degraded by any space-charge forces. With increased ion beam output from neutralized sources, circular and linear accelerators with much higher beam current and content of matter per beam line (vacuum tube) can be designed. Or, within the system of an existing accelerator, higher intensities of rare particles, such as highly charged heavy ions, can be transported from one section or sub-machine part to another. Efficient and economic ion source neutralization may replace the complex and expensive storage and cooling systems in the injection chains of big circular accelerators like the CERN Large Hadron Collider (LHC).

The technology for the compensation and neutralization of ion beams with low-energy electrons may benefit from a large range of available electron guns. The industrial production of electron source devices featuring the characteristic intensities, energies, and time structures suitable for ion beam neutralization is well advanced today.

\section{Space-charge dominated classical extraction schemes}

Ion beams are usually generated by the extraction of ions from a plasma source into vacuum through a gap loaded with an electric field. The extraction potential separates the accelerated ions from the plasma electrons, which are held back in the source. Conventional ion source extraction has been described in a large number of articles and monographs (see for example Refs. $[11,12]$ ). Classical ion beam extraction is always strongly intensity-limited by the space- 
charge of the slowly moving ions. The maximum ion current density $j_{i}$, which may be classically extracted from an ion source with an extraction potential $U_{a c}$ is approximately described by the Langmuir-Child law, which, in a plane gap of width $d$, is given by

$$
j_{i}=C_{\mathrm{LC}} \times U_{a c}^{3 / 2} / d^{2}
$$

with the Langmuir-Child constant

$$
C_{L C}=\left(\frac{4 \epsilon_{0}}{9}\right) \sqrt{\frac{2 e Z}{m_{i}}} .
$$

$\epsilon_{0}$ is the dielectric constant and $m_{i}$ and $Z$ are the mass and the charge number of the ions. A similar law is valid at low kinetic particle energy for the beam current in a circular or elliptic beam pipe. In the cylinder-symmetrical case, the azimuthal magnetic field of the beam and the distribution of the current density at the edge of the beam have to be taken into account. The $U_{a c}^{3 / 2}$-. Langmuir-Child law remains, however, qualitatively valid. If the ions have received a finite kinetic energy $e U_{0}$ already before leaving the anode and being accelerated in the extraction gap, the Langmuir-Child law can be approximately modified $[11,13]$ to

$$
j_{i}=\frac{4}{9} \epsilon_{0} \sqrt{\frac{2 e Z}{m_{i}}} \frac{1}{d^{2}}\left(\sqrt{U_{0}}+\sqrt{U_{0}+U_{a c}}\right)^{3} .
$$

The potential $U_{0}$ corresponds to the initial velocity of the ions. Hence a higher current density can be extracted from the anode compared to the density given in Eq. (1). In a drift space of length $d$ with $U_{a c}=0$, a current density of eight times the nominal Langmuir-Child current density (Eq. 1) determined by $U_{0}$ can be transmitted. Equation (3) can be usefully applied to a sequence of acceleration gaps, where $U_{0}=\sum_{\lambda} U_{\lambda}$ is the sum of all accelerating potential differences preceding the last gap with potential $U_{a c}$ and gap width $d$.

The nearer the beam intensity approaches the Langmuir-Child current density (Eq. 1), the stronger is the beam blow-up, which finally limits the output intensity. Another severe degradation caused by classical ion extraction, when approaching the Langmuir-Child limit, is the strong increase of the original source emittance $\epsilon_{s}$

$$
\epsilon_{s}=\sqrt{\frac{4 \pi}{m_{i} c^{2}}} \sqrt{\frac{k T \cdot I_{i}}{j_{i}}},
$$

where $k$ is the Boltzmann factor, $c$ the velocity of light, $T$ the temperature to which the emitting surface is heated, $I_{i}$ the total ion current, and $j_{i}$ the ion current density. 
The distance from the current density limit can be characterized at any time by the ratio of the real ion beam current density $j_{i}$, which flows in the axial direction, and the Langmuir-Child current density $j_{\mathrm{LC}}$. This ratio is called poissance $\Pi[11]$,

$$
\Pi=j_{i} / j_{\mathrm{LC}}
$$

which can be considered as the normalized perveance of the beam. The radial dimension of the beam envelope expands under the action of the space-charge forces from an initial beam radius $r_{b}$ after a distance $z$ to an output radius $r_{b o}$ according to Ref. [11]

$$
\int_{0}^{\sqrt{\ln \left(r_{b 0} / r_{b}\right)}} \exp \left(u^{2}\right) d u=\sqrt{\frac{\Pi}{18 \pi}} \times \frac{z}{r_{b}},
$$

not including the natural emittance contribution to the beam divergence. A beam with poissance $\Pi=1$ doubles its radius and increases its normalized emittance, also due to the additional angle spread, by more than one order of magnitude after a distance of approximately ten initial beam radii. Hence, strong compression of low-energy ion beams with external magnets or solenoids has disastrous and irrevocable effects on the quality of the beam.

The Langmuir-Child condition limits the output current, which can be extracted classically from an ion source to an extremely small value compared with the abundance of ions available in the source plasma. A tiny plasma layer of $1 \mu \mathrm{m} \times 30 \mu \mathrm{m} \times 30 \mu \mathrm{m}$ generated, for example, by an ultra-short laser pulse of a few $\mathrm{mJ}$ from a solid target surface may contain $10^{13}$ ions, whereas the single ion bunches of typical future collider accelerators will be only populated by $10^{7}$ to $10^{11}$ ions.

Classically extracted ion beams are usually transported further through vacuum before being post-accelerated in subsequent conventional accelerator structures to higher kinetic energy. Since, according to Eq. (1), the current density limit increases with $U_{a}^{3 / 2}$, the extraction potential must be as high as possible, and the drift space between the extraction gap and the next accelerating unit as short as possible, in order to limit ion losses and degradation of beam brightness. After the extraction gap of the ion source the beam is frequently guided by an axial magnetic field. If the ion beam travels through the magnetic field region without any divergence (Brillouin flow [12]), the magnetic field $B_{z}$ required for permitting the transport of ion current density $j_{i}$ is given by

$$
B_{z}^{2}=\frac{j_{i}}{\epsilon_{0}} \sqrt{\frac{m_{i}^{3}}{8 e^{3} Z^{3} U_{a c}}}
$$


Only beams with poissance $\Pi<1$ can be transported through a solenoid. In vacuum without magnetic field, a beam with $\Pi \geq 1$ and radius $r_{b}$ is virtually reflected after a stalling distance

$$
d_{0}=\sqrt{2} r_{b} / \sqrt{\Pi}
$$

which is even shorter inside a solenoidal field, since the Brillouin flow does not allow lateral expansion of the beam as in free vacuum. The radial expansion of an ion beam with $\Pi<1$ can be compensated with a strong solenoidal magnetic field, but the phase-space density is nevertheless diluted, since, in the longitudinal direction, the space-charge forces cannot be compensated. Hence, beam brightness is decreased and emittance increased, and therefore for an ion beam exiting the solenoid all subsequent conventional accelerating structures must be designed with a higher acceptance.

The only method available today by which one may improve beam quality, i.e. decreasing the emittance of charged particle beams and increasing their brightness, is the technique of beam accumulation in dedicated storage rings combined with the application of complex beam cooling. This complicated method is at present envisaged for the injection of heavy ion beams into the collider rings of future circular accelerators, such as the CERN LHC.

\section{Enhancement of extracted ion beam current with charge and current neutralization by electrons}

\subsection{Principles}

Full neutralization of low-energy ion beams may totally remove the tendency of beam blow-up and even yield perfect emittance preservation of beams with poissances $\Pi>1$. Whereas there is no chance of counteracting the strong space-charge forces of dense ion beams within a short distance by classical external electric or magnetic fields, the neutralization of the beam self-fields can be relatively easily achieved by electromagnetic interaction of the positively charged ion beam with particles of opposite polarity, i.e. electrons. Nature not only helps with space-charge neutralization, but, owing to the induction law, also with current neutralization. Hence, it is trivial to fully neutralize the space-charge and the current of a drifting ion beam of charge density e $Z n_{i}$ with electrons of charge density $e n_{e}$ travelling at the same velocity in the same direction. With full space-charge neutralization both charge densities must finally overlap and have to be equal

$$
n_{e}=Z n_{i} .
$$


For simultaneous current neutralization the ion $\left(j_{i}\right)$ and electron $\left(j_{e}\right)$ current densities must be oppositely directed and absolutely equal too,

$$
j_{e}+j_{i}=0
$$

With current neutralization at equal velocities the ion-electron recombination rate is not negligible [12]. It is therefore advantageous to maintain a small difference between absolute ion $\left(v_{i}\right)$ and electron $\left(v_{e}\right)$ velocities or to keep the electrons oscillating against the ions as in a plasma or in a solid crystal, whilst maintaining the equality of the average velocities. Independent of current neutralization, complete space-charge neutralization is always obtained, if at any time and location the expression

$$
j_{e}=j_{i} \cdot\left|v_{e} / v_{i}\right|
$$

is valid. Hence, a higher current of fast electrons is needed to compensate the space-charge of a slow ion beam current with lower intensity. Equation (11) also holds for counter-moving ion and electron beams, but in this case current neutralization is not possible at all. With space-charge neutralization the Langmuir-Child limit for the maximum extracted current density disappears. As much ion current and ion mass can be extracted as can be neutralized with the available electron beam current density, according to Eq. (11). In addition to the neutralized current density, a non-neutralized ion current density of the order of the Langmuir-Child intensity is always extracted. One may neutralize this part of the ion beam with additional electrons after the extraction gap, otherwise it will be partially lost.

Current self-neutralization is enforced by the induction law, according to which an induced electric field drives the neutralizing electrons into the direction of the ion beam flow

$$
E_{i n}=-\left(\frac{d\left(l \cdot I_{b}\right)}{d t}+r \cdot I_{b}\right)
$$

where $l, r$ are the inductance and the resistance per unit length of the beam, medium, and surroundings together. Equation (12) is valid in a beam pipe, when the induced wall current is much smaller than the induced current inside the beam and in the adjacent beam surroundings. When the compensating electron beam or cloud is 'in contact' with the ion beam, capacitive contributions to the loop impedance and displacement currents can be neglected in Eq. (12).

The neutralization of an ion beam with a co-travelling electron beam generates a completely force-free system. However, space-charge neutralization with 
counter-moving electrons leads to a pinching of the beam diameters. Since the ions move very slowly, their azimuthal magnetic field can be neglected in comparison with the azimuthal magnetic field of the electrons. The pinch radius $r_{p c}$ after neutralization is mainly determined by the emittance $\epsilon_{e}$ and the current $I_{e}$ of the electrons. When we assume full mutual charge neutralization, the beta-function $\bar{\beta}_{e}$ of the electron beam under the influence of its azimuthal magnetic self-field is given by

$$
\bar{\beta}_{e}=\pi \sqrt{\frac{2 \gamma_{e} m_{e}}{\mu_{0}}} \times \frac{c r_{p c}^{2} \sqrt{n_{e}}}{I_{e}},
$$

where $n_{e}, m_{e}$ and $\gamma_{e}$ are the number density, the mass, and the relativistic factor of the electrons. With the emittance of the electron beam,

$$
\epsilon_{e}=r_{p c}^{2} / \bar{\beta}_{e}
$$

the final radius $r_{p c}$ of the neutralized pinched channel follows as

$$
r_{p c}=\sqrt{\frac{2 \pi \gamma_{e} m_{e}}{\mu_{0} e}} \times \frac{c \epsilon_{e}}{\sqrt{v_{e} I_{e}}} .
$$

The channel geometry in which the extracted ion current propagates is controlled by the emittance, velocity, and the current intensity of the injected electron beam. The transverse and the longitudinal emittances of the ion beam are preserved inside the channel as long as the state of full space-charge neutralization is maintained. The additional non-neutralized part of the ion beam undergoes the same phase-space blow-up as a classically extracted ion beam. Equations (13-15) describe the end phase of full ion beam neutralization. The transition from the original state of non-neutralized ion and electron beams, where the condition in Eq. (9) has not yet been reached, to the state of full mutual space-charge neutralization is characterized by freely moving charges in strong electromagnetic fields, hence by phenomena that proceed on a very fast time scale compared with the average axial movement of the ion beam [14]. The same laws also hold for the process of current neutralization.

\subsection{Neutralization in the extraction gap by fast pulsed counter-moving elec- tron beams}

As shown in Section 2, several orders of magnitude of ion current density may be lost with conventional extraction during the transition from the source plasma to vacuum inside the extraction gap. For ion beam neutralization the extraction gap is the most difficult region, as the ions, if non-neutralized, 
start with the lowest velocity and the highest space-charge. The extraction gap is not only a drift space, but the ion beam has to be neutralized and is simultaneously accelerated. Current neutralization with co-travelling ion and electron beams is, therefore, excluded, as the electrons would be reflected into the source. Space-charge neutralization inside the extraction gap is achieved with counter-moving beams. Full neutralization occurs when taking into account Eq. (11) and the modification of the ratio $j_{e} / j_{i}$ along the longitudinal coordinate $z$ caused by the acceleration by $U_{a c}$ of both beams inside the gap of width $d[5]$

$$
\frac{j_{e}}{j_{i}}(z)=\sqrt{\frac{m_{i}}{Z m_{e}} \cdot \frac{U_{e 0}+\frac{U_{a c}}{d} \cdot(d-z)}{U_{i 0}+\frac{U_{a c}}{d} \cdot z}} .
$$

Here $e U_{i 0}$ and $e U_{e 0}$ are the entrance energies of the ions and the electrons going into either side of the extraction gap. Near the exit hole of the source the highest electron current density is required, hence, in this critical region the most stringent neutralization conditions have to be met. The extraction potential holds back all the plasma electrons in the source. Space charge neutralization is achieved exclusively by the injected and accelerated electron beam in accordance with Eq. (11). The maximum ion intensity, which can be extracted from the source, depends on the maximum electron density that can be injected towards the exit hole region. If the focusing angle of the incident electrons is approximately equal to the natural divergence angle of the neutralized ion beam exiting the extraction gap, then the ion beam will not suffer from emittance degradation. Although the acceleration of a straight electron beam by the extraction fields leads to a decrease of density, the external focusing and the emittance shrinking during acceleration can be used to compensate this dilution and to increase the electron density at the exit hole of the source to the desired value. Near the exit hole of the extraction gap the neutralization conditions are much less severe, as the ions have an increased velocity, whereas the electrons are still at their low injection energy.

The desired characteristics of the ion beam inside the extraction gap would favour the use of pulsed electron beams, especially when pulsed ion beams are envisaged. With pulsed electron beams much higher beam currents can be generated and a much higher ion current amplitude can be neutralized. With pulsed electron beams a temporal selection of fractions of the ion beam may be used to separate different ion charge states. Desired charge states are neutralized and, compared to conventional extraction, their intensity is enhanced, whereas undesired states are not neutralized and are lost due to space-charge blow-up. The total beam power of a d.c. electron beam of equal density would be much too high and would cause damage in the source, if high ion mass needs to be extracted. 
Figure 1 shows the set-up of ion neutralization experiments which have been carried out at CERN and which are described in Refs. [4-7]. The test set-up enabled ions to be extracted from a laser ion source with and without electron beam neutralization, mainly inside the extraction gap. The experiments showed that, in accordance with Eqs. (1),(3),(9) and (11), the ion current output from the source could be enhanced by about one order of magnitude with the available electron beam current (see Fig. 2 and Ref. [5]). Ferroelectricallygenerated pulsed hollow electron beam pulses [15] of about 50 ns FWHM length, with kinetic energies of a few $\mathrm{keV}$, with 1 to $5 \mathrm{~A}$ current amplitude and 10 to $30 \mathrm{~A} / \mathrm{cm}^{2}$ current density $[16,17]$ were sent in the opposite direction through the extraction gap into the source equipped with an $\mathrm{Al}$ target. When simply neutralizing the Langmuir-Child current density of $\mathrm{Al}^{1+}$ or $\mathrm{Al}^{2+}$ ions, the totally extracted current density measured immediately after the neutralization section was equal to $2 \times j_{\text {LC }}$, in accordance with the Langmuir-Child law. The enhancement factors were greater, the higher the chosen ion charge states (Fig. 2). Emittance measurements have not been made in the neutralization measurements reported in Refs. [4-7]. For the design of a realistic ion source enhancement scheme it is, however, obligatory to determine the source emittance $\epsilon_{s}$ at the entrance of the extraction gap (Eq. 4) as well as at the exit $\epsilon_{s}^{\prime}$, where

$$
\epsilon_{s}^{\prime}=\frac{\epsilon_{s} v_{i} \gamma_{i}}{v_{i} \gamma_{i}} \approx \epsilon_{s} v_{i} / v_{i}^{\prime}
$$

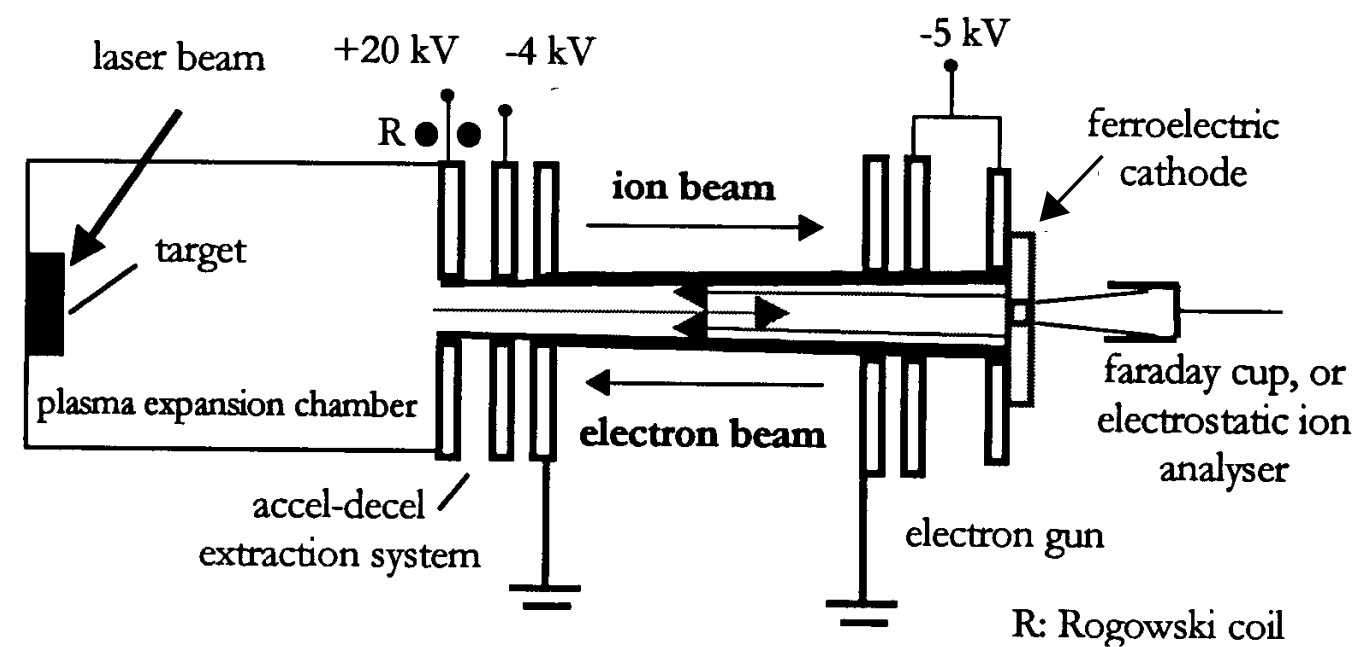

Fig. 1. Experimental set-up for ion beam neutralization in the extraction gap of a laser ion source [5]. 


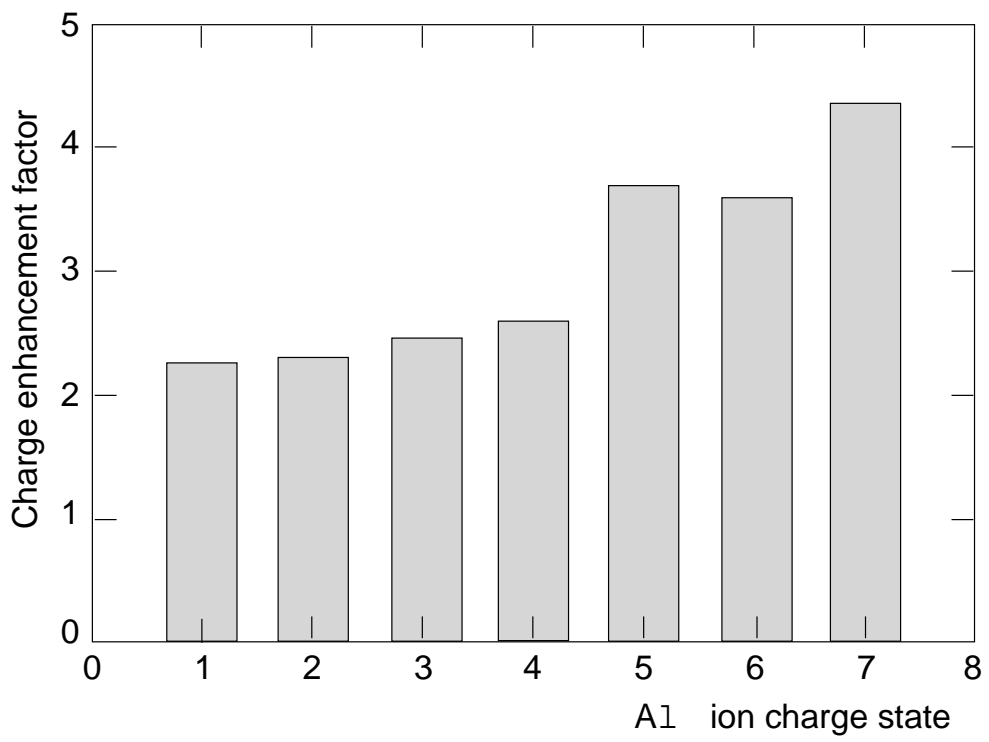

Fig. 2. Diagram of charge enhancement factors for neutralized $\mathrm{Al}$ ion beams with charge states from $1^{+}$to $7^{+}$.

with the ion velocities $v_{i}, v_{i}^{\prime}$ and the relativistic factors $\gamma_{i}, \gamma_{i}^{\prime}$ in front and after the extraction gap length. For classical ion extraction the initial ion velocity inside the source is of little importance, whereas a high initial axial ion velocity is favourable for the neutralized extraction, as long as the source emittance does not exceed the desired value. The emittances

$$
\epsilon_{s} \approx r_{b} \times \tilde{a}_{s} \text { and } \epsilon_{s}^{\prime} \approx r_{b}^{\prime} \times \tilde{a}_{s}^{\prime}
$$

can be approximately determined by measuring the ion beam radii $r_{b}, r_{b}^{\prime}$, and the divergence angles $\tilde{a}_{s}$ and $a_{s}^{\prime}$ on both sides of the extraction gap.

Ferroelectrically-driven electron guns are very convenient for ion beam neutralization inside the extraction gap. Strong enhancement of the ion source output current with thermionic electron sources has been reported in Refs. [18] and [19], although the extraction gap was only partially neutralized. In these experiments ion beam self-neutralization is also demonstrated with the thermionically-generated electrons, which are drawn by the space-charge field into the ion beam at the exit of the extraction gap of a laser ion source [19].

\subsection{Space-charge and current neutralization of drifting ion beams with slowly- pulsed co-travelling electron beams}

The neutralized ion beam leaving the extraction gap and entering the adjacent transport channel may have a poissance $\Pi$ well above 1 . It is, therefore, 
obligatory to maintain the neutralization of the ion beam and to avoid blowup. In the transition region between the exit hole of the extraction gap and the transport channel, for geometrical reasons it is usually not possible to apply a neutralization scheme with co-travelling electrons. As inside the extraction gap, counter-moving electron beams must be used instead. The matching conditions [Eqs. (9) and (11)] are, however, much easier to meet after the extraction gap than at the exit of the ion source. The ions have a higher speed and can be neutralized with much less electron current density than in the gap. By choosing the electron beam pulse length correctly, the ion charge state separation, which had been initiated in the extraction gap, can be completed in the transition region.

As soon as the geometry of the source exit and transport channel allows the injection of co-moving electron beams, further neutralization is very easy. After the neutralization process the co-moving electrons automatically fulfil the conditions of charge and current neutralization [Eqs. (9), (10) and (16)]. Comoving electrons are virtually attracted by the ion beam and continue to travel with the ion beam at the same average velocity. The requirements on electron guns, which can be applied for this type of neutralization, imply neither high brightness, nor confined beam geometry, but only total current, which must be equal or greater than the neutralized ion current. Owing to the initial attraction during the self-neutralization process, the electrons oscillate with respect to the ion beam.

In principle, such a fully charge- and current-neutralized ion beam can be transported over arbitrarily long distances with little particle loss and without danger of emittance degradation. Realistically the length of the transport channel has to be chosen according to the requirements of the post-acceleration equipment. The ion beam leaving a neutralization region has to be properly matched to the acceptance of the subsequent classical accelerator sections profiting from the higher Langmuir-Child limit valid at higher ion energy. Strong compression must imperatively also be avoided in the compensated stage. The drift tube has to be long enough, so that, by the natural divergence of the ion beam, its current density at the end is reduced so that $\Pi<1$, otherwise the beam degradation at the transition to the vacuum environment will be worse than without beam neutralization.

\subsection{De-neutralization of ion beams before injection into conventional accel- erator sections}

The danger of emittance blow-up at the exit of a neutralization section must be counteracted with adiabatic expansion of the ion beam in the neutralization region up to a beam radius, where space-charge becomes less significant 
$(\Pi<1)$. If the resulting beam cross-section is too large to fit into the next conventional accelerator element, the ion beam has to be sent through additional accelerating gaps with neutralization, before it can be released into a non-neutralized section. The electron beam parameters required for the neutralization of the ion beam in additional acceleration gaps are more relaxed the greater the entrance radius and velocity of the ion beam [Eq. (16)]. One may envisage a D.C. acceleration potential for a second gap, but if more than two gaps are necessary between the source and classical post-accelerating structure, pulsed acceleration fields are indispensable. The best available method is inductive acceleration. In this way, the whole low-energy beam transport system, except the ion source vessel, could be connected to ground potential.

Any conventional post-acceleration device requires complete de-neutralization of the ion beam, before it enters the classical acceleration scheme. For one D.C. extraction and one D.C. de-neutralization gap, the whole drift tube would have to be kept at the level of the de-neutralization potential. Owing to the small kinetic energy of the neutralizing electrons, pure de-neutralization can be achieved with a rather small potential. It is, however, favourable to apply an acceleration potential of the order of the extraction potential for the ion beam, which increases beam energy. Furthermore, the diameter and the real emittance of the ion beam are shrinking. Hence, the neutralized ion beam can arrive at the entrance of the de-neutralization section with a larger diameter than required by the acceptance of the post-acceleration unit.

\subsection{Neutralization of a complete ion injector for a conventional post- accelerating structure}

The application of ion beam neutralization is based on the availability of an ion source, which has, in accordance with the specifications of the overall system, the capability of producing a sufficiently strong ion current at a low enough source emittance $\epsilon_{s}$ [Eq. (4)], corresponding to a normalized emittance $\epsilon_{n}$, which is smaller than the final normalized target emittance of the system. If the conditions are not met, the application of ion beam neutralization is obsolete. Neutralization cannot improve beam quality, but, in the best case, could avoid further emittance blow-up. Hence the application of ion beam neutralization would be useless when the design specifications of a low-intensity ion beam accelerator can be accomplished with classical extraction and transport methods.

A convenient type of ion source for pulsed ion beam compensation is a laserdriven plasma source [20]. According to Eq. (4) a low source output emittance $\epsilon_{s}$ requires a low target surface temperature $T$ and high current density $j_{i}$, hence, a small laser spot. For generating high charge states Z, high laser power 
densities of the order of $\mathrm{TW} / \mathrm{cm}^{2}$ are essential, in order to establish a strong electromagnetic ionization field in the laser spot on the target. Therefore, the laser must feature a short pulse length (ps or fs) and a short wave length $(<1 \mu \mathrm{m})$ to be focused on a minimum spot size. The laser pulse energy determines the amount of material evaporated from the target and must be strictly minimized. Excess energy, for example if the laser pulse is too long, degrades the emittance [Eq. (4)] and produces unnecessary amounts of 'dead' plasma. After the ions have been generated on the target surface in a complicated way, they move on, fully neutralized by plasma electrons, with velocity $v_{i}$ inside the source chamber towards the extraction gap, where the ion beam acceleration and neutralization with externally injected electron beams has to start.

Figure 3 shows the principle of a possible neutralization scheme for a laser ion source with the aim of enhancing the extracted intensity beyond the Langmuir-Child limit and preserving the normalized ion source emittance until the 'end' of the neutralization section. When the source and the normalized emittances $\epsilon_{s}$ and $\epsilon_{n i}$ are smaller or equal to the required data, the remaining key parameters of the neutralized source are the exit hole radius of the plasma source $r_{p c}$ and the total ion current $I_{i}$, which the system has to deliver. The exit hole and neutralization channel radius is determined uniquely by the electron beam emittance $\epsilon_{e}$, velocity $v_{e}$ (energy), and current $I_{\epsilon}$ and can be calculated with Eq. (15). By combining Eqs. (9), (11), and (15) a total ion current of

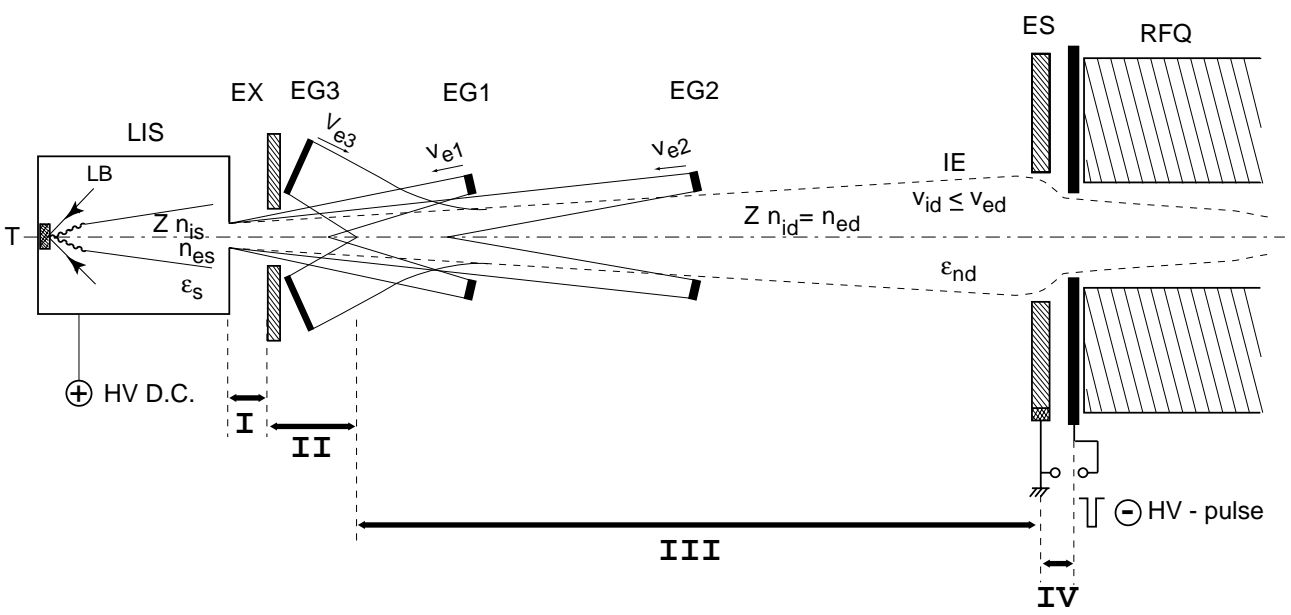

Fig. 3. Principle of an ion beam neutralization scheme for ion beam intensity enhancement and emittance preservation. LIS = laser ion source; LB = laser beam; $\mathrm{T}=$ target; $\mathrm{EX}=$ extraction gap; EG1,2,3 = electron guns; IE = ion beam envelope; $\mathrm{ES}=$ electrical separation gap; $\mathrm{RFQ}=$ radio frequency quadrupole; $\mathrm{Z}=$ charge state; $\epsilon_{s}=$ source emittance; $n_{i s}, n_{e s}=$ ion and electron densities in the source, $v_{e 1,2,3}=$ electron velocities from EG1,2,3; $\epsilon_{n d}=$ emittance in drift space, $n_{i d}, n_{e d}=$ ion and electron densities in drift space; $v_{i d}, v_{e d}=$ ion and electron velocities in drift space. 


$$
I_{i}=\frac{2 \pi^{2} m_{e} c^{2}}{\mu_{0} e} \times \frac{\gamma_{e} \epsilon_{e}^{2}}{v_{e}^{2} I_{e}} j_{e} \times v_{i}
$$

can be extracted in excess of the non-neutralized Langmuir-Child current. The total ion current $I_{i}$ is fully determined by the electron beam parameters $\gamma_{e}, v_{e}, j_{e}, \epsilon_{e}$, and by the initial velocity $v_{i}$ of the ions.

With a few modifications, the neutralization scheme of Fig. 3 can be adapted to any other ion source (including D.C. sources), fulfilling the emittance specifications. In any case, apart from the source proper, three stages of different types of neutralization are identified. The first stage (I in Fig. 3) is the extraction gap which is the most demanding part of the whole ion beam neutralization system. The electron gun EG1 has to provide fast intense electron pulses in the opposite direction to the ions, and be accurately focused into the exit hole of LIS in order to avoid HV breakdowns inside the extraction gap. The electron beam parameters of EG1, current density, energy and emittance, as well as the initial ion velocity, determine the extracted ion current according to Eq. (19). By synchronizing the laser and EG1 a pre-selection of the compensated charge-states can be achieved. Fast pulsed electron guns with cold field array emitter cathodes or ferroelectric cathodes seem convenient for stage I. In the second stage (II in Fig. 3), the ion beam is less dense and more energetic than in stage I. Therefore, similar, but less powerful, pulsed electron guns (EG2) are needed, which have to provide bursts of electron pulses moving in opposite directions into the transition region adjacent to the extraction gap. By proper synchronization with laser and electron guns (EG1), the charge state separation can be refined in the transition region. The third stage (III in Fig. 3) contains a simple low-intensity electron gun (EG3) which generates co-travelling electron pulses with only slightly higher charge, current, and pulse length, than the extracted ion beam pulse. In certain applications EG3 could even be a DC gun with modest intensity and power. The charge self-neutralization of the ion pulse along the drift channel by the co-travelling electron beams from gun EG3 is, therefore complemented by full current neutralization as described by Eqs. (9) and (10).

By natural divergence the ion beam diameter grows along the drift channel until the space-charge forces become negligible, if the ions are not neutralized. In order to match the ion beam to the subsequent classical accelerator section, for example an RFQ, the neutralizing electrons must be removed in the deneutralization stage IV (Fig. 3) and the ion beam diameter decreased by a pulsed accelerating $\mathrm{HV}$ pulse in front of the RFQ. The injected ion current amplitude and ion beam emittance controlled by the ion source and the ion beam geometry have to match the RFQ acceptance after de-neutralization. When a DC de-neutralization gap is used, the whole drift tube between the extraction gap and the RFQ has to be permanently kept at high voltage corresponding to the de-neutralization potential difference. 


\section{Applications}

With neutralization techniques new powerful ion beam sources can be built, which are able to produce intensities far beyond the Langmuir-Child limit, with negligible degradation of beam quality. Many applications involving high ion mass transport and acceleration, which cannot be achieved at all with conventional sources, may be designed with neutralization using low-energy electron beams. The technology of high-power guns for generating such electron beams can at present be considered as well advanced.

An important technical application of high ion mass beams in space technology are the ion thrusters for driving space vessels $[8,9]$. In principle, ion beam propulsion produces orders of magnitude more thrust with the same amount of ejected matter than conventional chemically-driven propulsion systems. However, today ion beam propulsion has, four important disadvantages:

a) The thrust of classically generated ion beams is so weak (tens of $\mathrm{mN}$ ) that ion thrusters carried by satellites can only be used for very small stabilizing or adjusting manipulations of the space vessel. Even an ion thruster with $1 \mathrm{MA} / 1 \mathrm{keV}$ corresponding to $1 \mathrm{GW}$ propulsion power has only a modest thrust $(<100 \mathrm{~N})$, compared with a classical chemical propulsion system.

b) Unlike classical propulsion, where the accelerated matter is at the same time the fuel for generating the thrust, the ion thruster needs additional energy for generating and accelerating the ion beam, as well as for generating of the neutralizing electrons.

c) The accelerated 'dead' ion mass does not contribute to power generation in the space vessel.

d) It is obligatory to fully neutralize the ejected ion beam, otherwise the space vessel is not accelerated at all.

Much more efficient ion beam thrusters could be built by applying neutralization with electron beams not only to the propagating ion beam after ejection into space, but by neutralizing the extraction gap of the ion source. Much higher ion mass could be accelerated in this way, but the necessary energy for acceleration would still have to be provided in an efficient way from a low-mass source. Today only a fission-driven energy supply would fulfil these requirements, and a combination of such a power source with a fully neutralized ion thruster would seem to be the optimum and feasible solution at this time.

Another application of high ion mass sources is the heating of magnetic confinement fusion plasmas in tokamaks by neutral beam injection. Such heating systems feature ion sources with emitting areas of many square metres. Although the ions are neutralized after exiting the extraction gap, the acceptance of the tokamak sets an upper limit the size of the ion source and for the 
injected neutral beam current. Much higher neutral beam currents and current densities may be injected into fusion reactors, if compact, high-brightness sources with extraction gap neutralization are developed and applied.

Other high ion mass applications are ion sources for accelerators driving spallation neutron sources, nuclear waste incineration systems, and acceleratordriven fission or inertial confinement fusion reactors. Because of the basic electromagnetic intensity limits it seems hopeless to efficiently generate, transport, and accelerate the ion mass required for these applications using conventional ion source and accelerator technology. Large conventional accelerators have, for example, in the past accelerated just a few grams of ion mass during several decades of operation. For the transmutation of hundreds of thousands of tons of nuclear waste material, clearly more powerful accelerators are needed. The combination of strong ion source neutralization schemes with inductive acceleration methods and with efficient target neutralization seems, at presents, to be the only way to build such high-intensity, high-power accelerators. Neutralization with electron beams would not be restricted to the ion source and the target areas, but would have to be extended over large sections of such machines.

Even in future accelerators for high-energy physics the ion mass to be accelerated per unit time is steadily growing compared with former classical accelerators. At the same time, better beam quality and brightness, e.g. lower emittance, are also required. Conventional technology tries to circumvent the poor performance of classical sources in medium ion mass applications by introducing multi-beam structures and accumulation and cooling rings for the ion beams at a certain level in the accelerator chain. Storage and cooling rings are, for example, also planned for the CERN Large Hadron Collider (LHC). Accumulation and ion beam cooling counteracts, to a certain extent, the degradation of normalized emittance $\epsilon_{n}$ by space-charge forces or other non-conservative forces when the beam is going through a classical accelerator. Not only, can the ion beam intensity be increased by storage, but cooling is the only known method by which large ion beam emittances can be reduced. However, storage and cooling rings are expensive and difficult to operate. The higher the ion beam energy and the worse the ion beam quality, the higher the cost of cooling. Accumulation and cooling must be introduced into the accelerator system at an energy level far above that of the ion source extraction energy, in order to make use of the rise of the intensity limit, which grows with $\beta^{3}=\left(v_{i} / c\right)^{3}$ (Eq. 1). Therefore, a considerable degradation of intensity and normalized emittance may occur in the accelerator part which precedes the storage and cooling rings. After the cooling phase, the non-neutralized ion beam is again restricted by the space-charge limit, though at a higher level. The application of fully neutralized ion sources may render storage and cooling rings obsolete, provided the normalized emittance of the ion source is smaller than the normalized emittance required in the accelerator, into which the ion beam has to be injected. 
Many other small and compact high-current accelerators may be equipped with neutralized sources, such as accelerators for medical purposes, for isotope production, and for ion implantation into semiconductors. In all cases, higher efficiency and lower costs may be achieved.

\section{Conclusions and outlook}

The neutralization of ion sources and ion transport channels removes the classical space-charge limits from ion extraction, which severely restrict the performance of conventional ion sources. The benefits of ion beam neutralization are the almost unlimited enhancement of the extracted ion intensity beyond the space-charge limit, and the preservation of ion beam quality represented by emittance and brightness. It has been shown that neutralization with intense, low-energy pulsed electron beams is a convenient way to neutralize ion beams under varying boundary conditions. At low ion mass applications, neutralized ion sources and accelerator sections can be matched to non-neutralized conventional accelerator units without major technical problems. Unlike radio frequency acceleration cavities only DC or switched induction acceleration gaps are suited to the acceleration of ion beams, which are simultaneously neutralized with pulsed electron beams. In principle, the construction of a complete and fully neutralized ion linac comes into reach.

With the introduction of neutralization techniques into ion generation, acceleration, and transport, the ambitious claims of accelerator technology, such as incinerating nuclear waste material, driving fission reactors and inertial confinement fusion plants, or constructing strong and efficient ion thrusters for space technology, may become a reality. Neutralization is also beneficial for ion accelerators, where less intensity, but high beam quality is the most important requirement. Injection of ion beams into large circular colliders, such as the future Large Hadron Collider at CERN, may be envisaged without making use of expensive and complex accumulation and cooling rings. The real technical application of neutralization techniques in any of the cited fields of science and technology will have to be preceded by dedicated pilot experiments in existing installations. Modest development efforts are necessary to adapt existing electron gun technology to the specific needs of the various neutralization schemes. 


\section{References}

[1] D. Gabor, Nature 160 (1974) 89.

[2] D.S. Prono et al., Survey of Initial Experiments on ATA Beam Dynamics, UCID-20264, LLNL (1984)

[3] R. Govil and W.P. Leemans, Experimental Observation of Return Current Effects in Passive Plasma Lenses, Paper presented at the Advanced Accelerator Concepts Workshop, 1998.

[4] U. Herleb, H. Riege, Proc. of the $11^{\text {th }}$ Int. Conf. on High Power Particle Beams, Beams '96, Prague, Czech. Rep. (1996) 1191.

[5] U. Herleb, Thesis, University of Erlangen (1997).

[6] H. Riege, U. Herleb, Proc. of the 11th Int. Conf. on High Power Particle Beams, Beams '96, Prague, Czech. Rep. (1996) 68.

[7] U. Herleb and H. Riege, Nucl. Instrum. Meth. A415 (1998) 464.

[8] M. Capacci and G. Noci, Rev. Sci. Instrum. 69 (2) (1998) 1.

[9] M. Capacci and G. Noci, US Patent No. 5,592,055.

[10] W. Möller et. al, Journal f. Oberflächentechnik, 37 / Nr.1 (1997), p. II.

[11] A.T. Forrester, Large Ion Beams (J. Wiley \& Sons, New York, 1988).

[12] I.C. Brown, The Physics and Technology of Ion Sources (J. Wiley \& Sons, New York, 1988).

[13] H. Rothe, W. Kleen, Elektronenröhren als End- und Sendeverstärker, Leipzig, Akad. Verl.- Ges. (1940).

[14] D.H. Whittum, A.M. Sessler, J.J. Steward and S.S. Yu, Particle Accelerators 34 (1990) 89.

[15] H. Riege, I. Boscolo, J. Handerek and U. Herleb, J. Appl. Phys., 84, Nr. 3 (1998) 1602.

[16] H. Gundel et. al., Ferroelectrics 100 (1989) 1.

[17] H. Gundel, J. Handerek, H. Riege, J. Appl. Phys. 69 (1991) 975.

[18] J. Ishikawa, F. Sano, T. Takagi, J. Appl. Phys. 53 (9) (1982) 6018.

[19] S.A. Kondrashev, B. Yu. Sharkov, J. Collier, T.R. Sherwood, Internal report ITEP 73-94, Moscow (1994).

[20] GSI-98-10 Report (1998) 18. 